\title{
An inexpensive microcomputer-based image digitizer
}

\author{
WILLIAM JAMES TIETJEN \\ Department of Biology, Georgia College, Milledgeville, Georgia 31061
}

\begin{abstract}
An inexpensive (under $\$ 600$ ) microcomputer-controlled image analysis system that uses commercially available components is described. Both programming and construction details are provided, as well as a discussion of the utility of the apparatus in studies of animal behavior. The system has been used to record position preferences and activity in animal groupings of various size. Simply by changing the distance between the target and the camera, animals and/or arenas of differing size can be monitored.
\end{abstract}

This paper describes an inexpensive microcomputer interface that allows simple image analysis. The system is adaptable to microcomputers having an input/output port, video display, and as little as 512 bytes of memory. The apparatus was designed to monitor position and movements in groups of the social spider Mallos gregalis. For M. gregalis, the system has been used to monitor activity, web structure, and animal positions (Tietjen, in press). It is nonintrusive and adaptable to a variety of experimental applications, including monitoring animals or enclosures of differing size, as well as animal groups of various sizes. The apparatus has been used, for example, to monitor the activity and positions of houseflies (Musca domestica) in an olfactometer and the rhythms of movement in cultured chicken embryos (Gallus domesticus).

\section{PRINCIPLE OF ORATION}

The computer is an RCA CDP-1802-based microcomputer system with $8 \mathrm{~K}$ bytes of memory (Nectronics, Ltd., Litchfield Road, New Milford, Connecticut 06776). This microcomputer was chosen because (1) complementary metal oxide semiconductor (CMOS) architecture has low power requirements and high noise immunity, permitting noncritical component layout, and (2) graphics display capability is included in the basic computer, allowing generation of a bit-mapped display used in camera set-up. A possible limitation of this system for some users may be the relatively slow processor speed, with a typical instruction execution time of about 9 microsec. Nonetheless, over $200 \mathrm{frames} / \mathrm{sec}$ can be generated by the apparatus, and speed was not a limiting factor in the current application.

This research was supported by National Science Foundation Grant BNS 79-10186 to W. J. Tietjen and was conducted in the laboratories of the North Carolina Department of Mental Health and Georgia College Department of Biology. I am grateful to P. N. Witt and K. S. Saladin for many helpful discussions during the course of this research.
The camera is a commercially available image digitizer (Type 511, Periphicon, P.O. Box 324, Beaverton, Oregon 97005) and is provided with an f $1.913-\mathrm{mm}$ lens focusing from $.2 \mathrm{~m}$ to infinity. Spectral response ranges from infrared to near UV, with peak sensitivity at about $960 \mathrm{~nm}$. The photosensitive array is arranged as a square matrix of 32 by 32 picture elements $(1,024$ pixels $)$, which, after exposure, represent a binary (light vs. dark) image of the scene within the camera's field of view. The camera input/output lines present one 74LS load to the computer, are TTL compatible, and are accessed by means of a six-contact edge card. Power requirements are $+5 \mathrm{~V}, 75 \mathrm{~mA}\left(\mathrm{~V}_{\mathrm{cc}}\right),-9$ to $-12 \mathrm{~V}, 75 \mathrm{~mA}\left(\mathrm{~V}_{\mathrm{bb}}\right)$, and system ground. Connections to the computer are by means of two input lines (clock and reset) and a serial data output line.

To quantize a picture, the computer first sends a reset pulse to the camera, followed by 1,024 clock pulses to initialize each element in the photosensitive array. A solftware loop is then used to control exposure time and is followed by a second set of 1,024 clock pulses, during the execution of which data are received by the camera. Each clock cycle causes the camera to step through the array and transmit one pixel of intensity information to the camera. The data are then formatted for display and/or analyzed and stored in memory.

Two input modes to the computer are available and are switch selectable: The serial mode is enabled by placing three switches in their "s" positions; the parallel mode is enabled by selecting the "p" positions (Figure 1). Components common to both modes include CMOS noninverting buffers, a voltage adaptor, and input/output ports. The buffers provide a high input impedance and increased noise immunity. A multivoltage adaptor board (Jameco Electronics, 1355 Shoreway Road, Belmont. California 94002) converts $+5 \mathrm{~V}$ from the computer to a ripple-free $-12 \mathrm{~V} 160-\mathrm{mA}$ output used to bias the camera. A potentiometer in series with the bias line permits adjustment of contrast. The output port is used to strobe a pulse to the reset line 


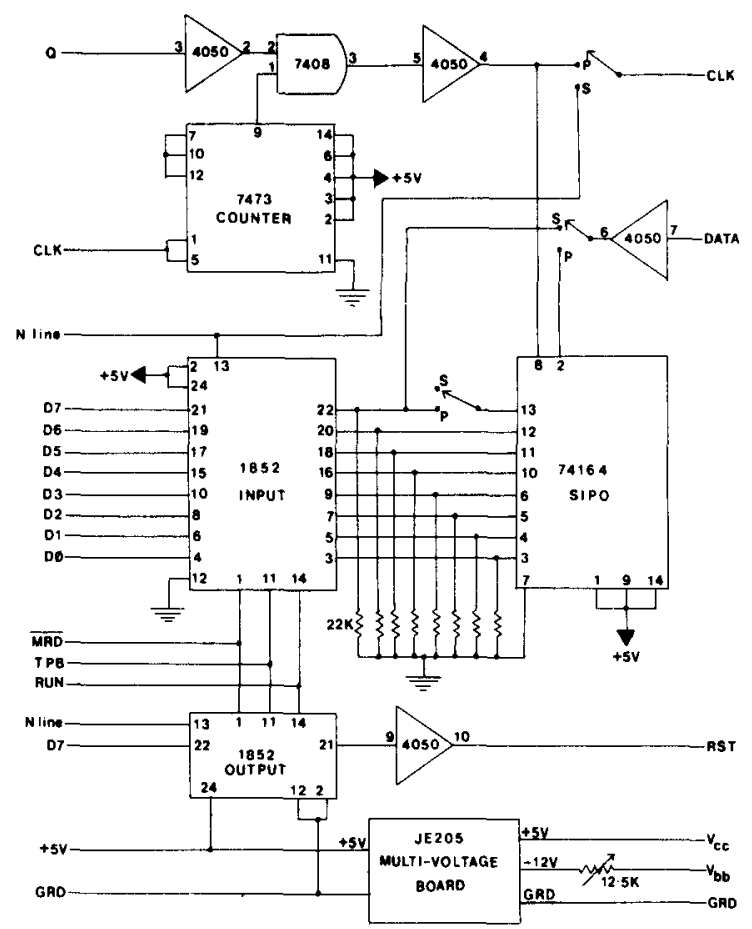

Figure 1. Serial and parallel interface electronics. Bus signals available from the 1802-based computer are shown on the left; signals connected directly to the camera are shown on the right. The serial input mode is selected by placing all switches in the " $s$ " position; parallel input is selected by the " $P$ " position.

of the camera, and the input port receives either serial or parallel data from the camera, depending on the switch positions.

In the serial input mode, 1,024 input-port instructions are performed by the program. A device-select pulse ( $\mathrm{N}$ line) provides the clock to the camera, and binary data are read from the input port during this pulse. For a computer clock speed of $1.8 \mathrm{MHz}$, minimum image integration time is about $18 \mathrm{msec}$. Integration times greater than $20 \mathrm{msec}$ require the full bias voltage of $-12 \mathrm{~V}$; otherwise, spurious artifacts may appear. Using the maximum contrast, however, will allow integration times of greater than $150 \mathrm{msec}$.

For the parallel mode, the system clock is routed through a divide-by-four counter to provide a camera clock of approximately $447 \mathrm{KHz}$. The minimum integration time at this frequency is 2 msec. The clock output to the camera is controlled by means of a programmable serial data line from the computer (Q). Data from the camera are sent to a serial-in/parallel-out shift register, and the parallel data are available to the computer bus through the input port.

Each input mode has advantages and disadvantages. The serial mode requires a $1 \mathrm{~K}$-byte buffer in memory for the camera data. In addition to being more memory intensive than the parallel mode (128-byte buffer), the serial mode is considerably slower. If the data are to be formatted for display, then the single-bit data in the frame buffer must be packed into an 8-bit byte. This process results in some image flicker on the television monitor. For most applications, however, the speed of the serial mode is sufficient.

The serial mode has the advantage of being relatively insensitive to slight differences in light intensity within the camera's field of view. The parallel mode, on the other hand, requires careful adjustment of illumination to sustain contrast within the recorded area. A second potential disadvantage of the parallel mode relates to random fluctuations in image position at the beginning of each experimental series. These fluctuations occur because the counter controlling the clock line cannot be preset to zero. Once an experiment begins, however, the image does not shift, since the counter remains in step with the program. The resulting shifts can then be corrected during analysis of data.

A detailed description of illumination techniques is presented elsewhere (Tietjen, in press) and can be obtained by writing to the author. Briefly, recording chambers containing the experimental animals are 52.2-cc petri dishes placed on a clear Plexiglas base. Diffused light is transmitted through the bottom of the chambers, such that the animals within are recorded by the overhead camera as black dots on a white background. Using these techniques, animal groups of 1-20 individuals have been recorded.

Programs used to control the camera, format data, and perform simple image analysis are written in a modular framework. ${ }^{1}$ Although such designs often lead to inefficiencies in execution speed or memory utilization, the program modules are easily linked into a largeapplication program. In addition, since each module has been tested, development time for larger programs is reduced.

The camera-driver program provides all signals necessary to control the camera. Exposure time, number of frames per second, and exit to a data storage routine are controlled by the operator from the keyboard. Data are usually stored on magnetic tape; however, options exist for transmitting the data to a second computer or printer. The camera-driver program also formats the data and contains the monitor-driver routine used to display the picture.

Other functions or preprocessing of data are programmed by linking a variety of modules to the above camera-drive routine. Identification of individual animals and/or areas within the chambers is accomplished by means of a lightemitting diode (LED) connected to the programmable $Q$ line. In use, the LED is held over the animal and an identification number is entered by means of the computer keypad. The computer then labels the indicated position in memory with the identification number .

The animal's use of space within the chamber is recorded by keeping a running count of positions occupied by animals over time. Up to 65,536 frames of data can be collected to record the use of space. 
These data provide a measure of preferences for various positions within the container.

Activity of the experimental animals is recorded by comparing the occupied positions in two consecutive pictures. A count of the number of movements that occurred between frames with respect to time are stored in memory. With $8 \mathrm{~K}$ bytes of memory, 5,120 frames can be analyzed, with up to 255 possible moves occurring between pictures before overflow takes place. The activity and use-of-space data may be collected concurrently by linking the two programs to a cameradriver routine.

A gray-level representation of the image is constructed by starting at the minimum exposure time and recording in memory the positions of those pixels activated by light. The exposure time is then increased, usually in $.03-\mathrm{msec}$ increments, 1,024 times. Each iteration results in darker areas in the field of view being recorded. This results in a gray-level representation of the image in 1,024 levels at each of the positions.

The CDP 1802 computer is not well suited for statistical analysis of data. Image data are therefore transmitted to an Ohio Scientific C1-P microcomputer for analysis (Ohio Scientific, 1333 South Chillicothe Road, Aurora, Ohio 44202) (see Footnote 1). The C1-P will support either a 6.5- or 9.5-digit BASIC. Because of this relatively low precision and the large data sets involved, most analyses are nonparametric.

\section{PRESENT STATUS AND FUTURE DEVELOPMENT}

In addition to the above functions, software packages are being written to record the incidence of social contact, velocity of movement, and recognition of individual animals within groups over long periods of time. Hardware enhancements currently under construction include the addition of $36 \mathrm{~K}$ bytes of memory, an arithmetic processor, and a color display to aid in the interpretation of data. Consideration is being given to using a second camera orthogonal to the first to allow the recording of movements in three dimensions.

The apparatus is both inexpensive and simple to build. The high noise immunity of the CMOS circuitry and relatively low clock speeds allow noncritical component placement and point-to-point wiring techniques. The total cost of an $8 \mathrm{~K}$ byte imaging system, including a magnetic recorder and television monitor, is less than $\$ 600$. If another computer is not available for data analysis, an additional $\$ 400-\$ 1,200$ must be allocated. Alternately, the CDP 1802 computer can be expanded to support a high-level language such as BASIC, and all data analyses can be performed within the one computer. Such a route would cost an additional $\$ 200$ $\$ 300$, depending on options chosen. The major disadvantage of this technique is the long period of time needed for the analyses compared with that needed by the Cl-P computer.
Although the system does not have the patternrecognition capabilities of the apparatus described by Kernan, Higby, Hopper, Cunningham, Lloyd, and Reiter (1980), it is effective for collecting data related to animal position and movement at a fraction of the cost. It is unlikely that this system could be programmed to recognize more than the simplest patterns because of the relatively low resolution of the camera and the slow processor speed, which hampers real-time analysis of large data sets.

Careful set-up and regulation of light intensity is an absolute necessity, as the camera is very sensitive to changes in light level. In addition, temperature of the apparatus and the target should remain relatively constant, because of the camera's peak sensitivity in the infrared; temperature variations greater than $5^{\circ} \mathrm{C}$ are recorded as changes in light intensity. Such factors may limit the camera's application if large arenas are used or light is reflected from the target rather than transmitted through it. The chambers used during the experiments should also provide a relatively homogeneous image to the camera for similar reasons.

Thus far, data have been collected on the effect of group size on animal activity, use of web space, and the rate of growth of $M$. gregalis nests. The response of houseflies has also been recorded in olfactometers using $M$. gregalis webs and various control odors as stimuli. Simply by adjusting the distance between the camera and the target, various arena and animal sizes can be recorded. In most of the experiments with $M$. gregalis, the camera is adjusted such that a spider occupies one element of the array. This arrangement provides maximum resolution as to animal position. By moving the camera closer, movements such as grooming behavior can be recorded and analyzed.

The sensitivity of the camera in the infrared wavelengths allows the experimenter to use an illumination source that is outside the visual range of many animals. Since the camera is controlled by the computer, a reference point in the recorded area would allow the computer to adjust exposure times automatically over a range of light intensities. This technique would permit a natural photoperiod during an experimental sequence. An additional advantage of computer control is that the large data sets generated by this apparatus are already in a form that can be accepted as input to another computer, thus eliminating hand coding of data.

\section{REFERENCES}

Kernan, W. J., Higby, W. J., Hopper, D. L., Cunningham, W., Lloyd, W. E., \& Reiter, L. Pattern recognition of behavioral events in the nonhuman primate. Behavior Research Methods \& Instrumentation, 1980, 14, 524-534.

TIETJEN, W. J. Sanitary behavior by the social spider Mallos gregalis (Dictynidae): Distribution of excreta as related to web density and animal movements. Psyche, in press. 


\section{NOTE}

1. Machine language listing with comments are available from this author. Work is currently in progress to adapt this apparatus to the Ohio Scientific C1-P computer. Programs and hardware developed for the C1.P system should be adaptable to any 6502-based microcomputer.

(Received for publication April 24, 1981; accepted April 28, 1981.) 